\title{
Quintessence and Cosmic Acceleration
}

\author{
M. K. Mak* \\ Department of Physics, The Hong Kong University of Science and Technology,Clear Water Bay, Hong Kong, P. R. China. \\ T. Harko ${ }^{\dagger}$ \\ Department of Physics, The University of Hong Kong, Pokfulam Road, Hong Kong, P. R. China.
}

\begin{abstract}
A cosmological model with perfect fluid and self-interacting quintessence field is considered in the framework of the spatially flat Friedmann-Robertson-Walker (FRW) geometry. By assuming that all physical quantities depend on the volume scale factor of the Universe, the general solution of the gravitational field equations can be expressed in an exact parametric form. The quintessence field is a free parameter. With an appropriate choice of the scalar field a class of exact solutions is obtained, with an exponential type scalar field potential fixed via the gravitational field equations. The general physical behavior of the model is consistent with the recent cosmological scenario favored by supernova type Ia observations, indicating an accelerated expansion of the Universe.
\end{abstract}

\section{INTRODUCTION}

Measurements of the cosmic microwave background, the mass power spectrum and of the luminosity-red shift relation observed for Type Ia supernovae with redshift up to about $z \lesssim 1$ provided evidence that we may live in a low mass-density Universe, with the contribution of the non-relativistic matter (baryonic plus dark) to the total energy density of the Universe of the order of $\Omega_{m} \sim 0.3$ [1]- [3]. The value of $\Omega_{m}$ is significantly less than unity [4] and consequently either the Universe is open or there is some additional energy density $\rho$ sufficient to reach the value $\Omega_{\text {total }}=1$, predicted by inflationary theory. The missing energy should possess negative pressure $p$ and equation of state $\gamma=\frac{p}{\rho}<0$. Observations also show that the deceleration parameter of the Universe $q$ is in the range $-1 \leq q<0$, and the present-day Universe undergoes an accelerated expansionary evolution.

Several physical models have been proposed to give a consistent physical interpretation to these observational facts. One candidate for the missing energy is vacuum energy density or cosmological constant $\Lambda$, satisfying the equation of state $p=-\rho$ [5]. According to this cosmological model the present-day Universe consists of a mixture of vacuum energy and cold dark matter. The conditions under which the dynamics of a self-interacting Brans-Dicke (BD) field can account for the accelerated expansion of the Universe have been considered in [6]. In this scenario accelerated expanding solutions can be obtained with a quadratic self-coupling of the BD field and a negative coupling constant $\omega$. But a cosmic fluid (pressureless and with pressure) obeying a perfect fluid type equation of state cannot support the acceleration in the BD theory.

Another possibilities are cosmologies based on a mixture of cold dark matter and quintessence, a slowly-varying, spatially inhomogeneous component [7]. An example of implementation of the idea of quintessence is the suggestion that it is the energy associated with a scalar field $\phi$ with self-interaction potential $U(\phi)$. If the potential energy density is greater than the kinetic one, then the pressure $p_{\phi}=\frac{1}{2} \dot{\phi}^{2}-U(\phi)$ associated to the $\phi$-field is negative. Quintessencial cosmological models have been recently intensively investigated in the physical literature [8]. For quintessence, the equation of state defined by $\gamma_{\phi}=\frac{p_{\phi}}{\rho_{\phi}}$ is subject to the constraint $-1 \leq \gamma_{\phi} \leq-0.6$.

But all these scenarios present two major difficulties [9]. The first one is the fine-tunning problem: why is the missing energy density today so small compared to typical particle physics scales? If today $\Omega_{m} \sim 0.3$, then the missing energy density is of the order of $10^{-47} \mathrm{GeV}$, which appears to require the introduction of a new mass scale of 14 orders of magnitude smaller than the electroweak scale. The second problem is the "cosmic coincidence" problem: since the missing energy density and the matter density decrease at different rates as a result of the expansion of the Universe, their ratio must be set to a specific, infinitesimal value in the very early Universe in order for the two densities to nearly coincide today. In order to solve these problems a form of the quintessence field, called "tracker fields", has been introduced, in which the tracker field $\phi$ rolls down the potential $U(\phi)$ according to an attractor-like solution to the equations of motion [9].

*E-mail:mkmak@vtc.edu.hk

${ }^{\dagger}$ E-mail: tcharko@hkusua.hku.hk 
Most of the studied quintessence models involve minimally coupled scalar fields with different potentials. One of the must studied is the simple exponential potential. But in this case the energy density is not enough to close to $\Omega=1$ the total energy density of the Universe. To solve the cosmic quintessence problem inverse power law potentials have been considered [10]. For this potential the predicted value of $\gamma_{\phi}$ is not in good agreement with observations. The implications for the cosmological evolution of potentials, which asymptotically behaves like the inverse power law or exponential potentials, of the form $U_{0}[\cosh (\lambda \phi)-1]^{p}[11]$ or $U_{0}\left[\sinh \left(\alpha \sqrt{k_{0}} \Delta \phi\right)\right]^{\beta}$ [12], have also been studied.

The nature of the scalar field potential compatible with a power law expansion in a self-interacting BD cosmology with a perfect fluid background has been analyzed in [13]. The form of the cosmic potential has been reconstructed for a minimally coupled quintessence field, in [14], by using observational data, from the expression of the luminosity distance $d_{L}(z)$ as function of the redshift $z$. General cosmological models with non-minimal coupling of the scalar field have been considered in [15].

It is the purpose of the present Letter to consider some exact classes of solutions of the gravitational field equations for a spatially flat FRW Universe composed from a mixture of matter and quintessence field. By assuming that all physical quantities depend on the volume scale factor of the Universe only, the general solution of the field equations can be expressed in an exact parametric form, with the volume scale factor taken as parameter and the quintessence field $\phi$ as a free parameter. By appropriately choosing the mathematical form of $\phi$ a cosmological model is obtained which incorporates the basic observational properties of the present day Universe.

The present Letter is organized as follows. The basic equations of the model and the general solutions are obtained in Section II. The general solution to the field equations corresponding to dust matter is presented in Section III. In Section IV we discuss and conclude our results.

\section{CLASSES OF GENERAL SOLUTIONS OF FIELD EQUATIONS WITH MATTER AND QUINTESSENCE}

We assume that the matter content of the Universe is composed of a perfect fluid with the pressure $p_{m}$ and density $\rho_{m}$, obeying a barotropic equation of state of the form $p_{m}=(\gamma-1) \rho_{m}, 1 \leq \gamma \leq 2$ and a scalar field $\phi$ as the source of the quintessence matter, with pressure $p_{\phi}=\frac{1}{2} \dot{\phi}^{2}-U(\phi)$ and energy density $\rho_{\phi}=\frac{1}{2} \dot{\phi}^{2}+U(\phi) . U(\phi)$ is the potential energy of the quintessence field.

The line element for a spatially flat FRW cosmological model takes the form

$$
d s^{2}=d t^{2}-a^{2}(t)\left(d x^{2}+d y^{2}+d z^{2}\right) .
$$

In the present paper we use units so that $8 \pi G=c=1$.

By introducing the Hubble function $H=\frac{\dot{a}}{a}=\frac{\dot{V}}{3 V}$ (where a $\operatorname{dot} \cdot=\frac{d}{d t}$ ) and $V(t)=\left(\frac{a(t)}{a_{0}}\right)^{3}, a_{0}=$ constant $>0$, the gravitational field equations and the equation of motion of the quintessence field are:

$$
3 H^{2}=\rho_{m}+\rho_{\phi}, 2 \dot{H}+3 H^{2}=-(\gamma-1) \rho_{m}-p_{\phi}, \ddot{\phi}+3 H \dot{\phi}=-\frac{d U(\phi)}{d \phi} .
$$

The energy conservation equation of the baryonic matter, which follows from the Bianchi identity gives $\dot{\rho}_{m}+$ $3 H \gamma \rho_{m}=0$.

Therefore the time evolution of the energy density of the matter component of the Universe, obeying a barotropic equation of state, is given by $\rho_{m}=\rho_{0} V^{-\gamma}$, where $\rho_{0}$ is a positive constant of integration.

The observationally important physical quantity, the deceleration parameter $q$ is defined according to $q=\frac{d H^{-1}}{d t}-1$. The sign of the deceleration parameter indicates whether the cosmological model accelerates or decelerates. The positive sign corresponds to decelerating models whereas the negative sign indicates accelerated expansion.

By substracting Eqs. (2) we obtain

$$
2 \dot{H}+\dot{\phi}^{2}+\gamma \rho_{m}=0 .
$$

With the use of the definition of the Hubble function and of Eqs. (2), Eq. (3) can be transformed to the form

$$
\frac{\ddot{V}}{V}-\left(\frac{\dot{V}}{V}\right)^{2}+\frac{3}{2} \gamma \rho_{0} V^{-\gamma}+\frac{3}{2} \dot{\phi}^{2}=0 .
$$


By means of the substitution $\dot{V}=u$ and $\ddot{V}=u \frac{d u}{d V}=\frac{1}{2} \frac{d}{d V} u^{2}$ we obtain the following first order linear differential equation in the variable $u^{2}$ describing the dynamics of the Universe for a mixture of matter and scalar quintessence field:

$$
\frac{d\left(u^{2}\right)}{d V}+\left[3 V\left(\frac{d \phi}{d V}\right)^{2}-\frac{2}{V}\right] u^{2}=-3 \gamma \rho_{0} V^{1-\gamma} .
$$

On integration, we obtain the solution of Eq. (5) in the form

$$
u^{2}=V^{2} e^{-3 \int V\left(\frac{d \phi}{d V}\right)^{2} d V}\left[g-3 \gamma \rho_{0} \int V^{-1-\gamma} e^{3 \int V\left(\frac{d \phi}{d V}\right)^{2} d V} d V\right]
$$

where $g$ is a constant of integration. On further integration, we obtain the general solution of Eq. (4):

$$
t-t_{0}=\int \frac{e^{\frac{3}{2} \int V\left(\frac{d \phi}{d V}\right)^{2} d V}}{V \sqrt{g-3 \gamma \rho_{0} \int V^{-1-\gamma} e^{3 \int V\left(\frac{d \phi}{d V}\right)^{2} d V} d V}} d V .
$$

where $t_{0}$ is a constant of integration.

Therefore the general solution of the Einstein's gravitational field equations with matter and quintessence field can be expressed in the following exact parametric form, with $V>0$ taken as parameter:

$$
\begin{aligned}
& \phi=\phi(V), a=a_{0} V^{\frac{1}{3}}, \rho_{m}=\rho_{0} V^{-\gamma}, p_{m}=(\gamma-1) \rho_{m}, \\
& U(V)=\frac{V^{2}\left[g-3 \gamma \rho_{0} \int V^{-1-\gamma} e^{3 \int V\left(\frac{d \phi}{d V}\right)^{2} d V} d V\right]\left[\frac{1}{3} V^{-2}-\frac{1}{2}\left(\frac{d \phi}{d V}\right)^{2}\right]}{e^{3 \int V\left(\frac{d \phi}{d V}\right)^{2} d V}}-\rho_{0} V^{-\gamma}, \\
& \rho_{\phi}(V)=\frac{1}{3} \frac{\left[g-3 \gamma \rho_{0} \int V^{-1-\gamma} e^{3 \int V\left(\frac{d \phi}{d V}\right)^{2} d V} d V\right]}{e^{3 \int V\left(\frac{d \phi}{d V}\right)^{2} d V}}-\rho_{0} V^{-\gamma}, \\
& p_{\phi}(V)=\frac{V^{2}\left[g-3 \gamma \rho_{0} \int V^{-1-\gamma} e^{3 \int V\left(\frac{d \phi}{d V}\right)^{2} d V} d V\right]\left[\left(\frac{d \phi}{d V}\right)^{2}-\frac{1}{3} V^{-2}\right]}{e^{3 \int V\left(\frac{d \phi}{d V}\right)^{2} d V}}+\rho_{0} V^{-\gamma}, \\
& q(V)=\frac{9}{2}\left\{V^{2}\left(\frac{d \phi}{d V}\right)^{2}+\frac{\gamma \rho_{0} e^{3 \int V\left(\frac{d \phi}{d V}\right)^{2} d V}}{V^{\gamma}\left[g-3 \gamma \rho_{0} \int V^{-1-\gamma} e^{3 \int V\left(\frac{d \phi}{d V}\right)^{2} d V} d V\right]}\right\}-1 .
\end{aligned}
$$

Classes of exact solutions of the field equations can be easily generated by the appropriate choice of the scalar field $\phi$ as an arbitray function of volume scale factor $V$. Via the gravitational field equations the choice of the quintessence field also fixes the mathematical form of the potential.

\section{COSMOLOGICAL DUST SOLUTION WITH QUINTESSENCE FIELD}

Assuming that at the present period the Universe is cold baryonic matter dominated with negligible thermodynamic pressure, we can put in the field equations $p_{m}=0$, or, equivalently, we choose $\gamma=1$.

In order to have a clear physical interpretation of the solutions, we choose the scalar field $\phi$ in the form $\phi=\sqrt{n} \ln V$, where $n$ is a positive constant.

With this choice of the scalar field $\phi$ we obtain a solution of the Einstein gravitational field equations with dust matter and quintessence, which can be expressed in the following exact parametric form: 


$$
\begin{gathered}
t-t_{0}=\int \frac{V^{\frac{3 n}{2}-1}}{\sqrt{g+\frac{3 \rho_{0}}{1-3 n} V^{3 n-1}}} d V, a=a_{0} e^{\frac{\phi}{3 \sqrt{n}}}, \rho_{m}=\frac{\rho_{0}}{V}, \\
U(V)=\left(\frac{1}{3}-\frac{n}{2}\right) F(V)-\rho_{0} V^{-1}, \rho_{\phi}(V)=\frac{1}{3} F(V)-\rho_{0} V^{-1}, \\
p_{\phi}(V)=\left(n-\frac{1}{3}\right) F(V)+\rho_{0} V^{-1}, q(V)=\frac{9}{2}\left[n+\frac{\rho_{0}}{V F(V)}\right]-1,
\end{gathered}
$$

where $F(V)=g V^{-3 n}+\frac{3 \rho_{0}}{1-3 n} V^{-1}$.

The time transformation can be evaluated and expressed in terms of hypergeometric functions.

In order that the Universe undergoes an accelerating phase, it is necessary that $q<0$. By imposing this condition and with the use of Eqs. (14) and (15), we obtain the following constraint on the parameter $n$ :

$$
n<\frac{2 \frac{\rho_{\phi}}{\rho_{m}}-1}{9\left(\frac{\rho_{\phi}}{\rho_{m}}+1\right)} .
$$

By assuming a ratio of the quintessence field energy density and matter energy density of the order of $\frac{\rho_{\phi}}{\rho_{m}} \approx \frac{7}{3}[1-3]$, we find for the parameter $n$ the constraint $n<0.12$. By assuming for the ratio $\frac{\rho_{\phi}}{\rho_{m}}$ a value of $4\left(\rho_{\phi} \propto 0.8, \rho_{m} \propto 0.2\right)$, we obtain for $n$ the condition $n<0.155$.

The form of the quintessence field potential follows from Eqs. (13) and (14) and is given, as a function of the field by

$$
U(\phi)=3 \rho_{0} \frac{n}{2(1-3 n)} e^{-\frac{\phi}{\sqrt{n}}}+g\left(\frac{1}{3}-\frac{n}{2}\right) e^{-3 \sqrt{n} \phi} .
$$

The dependence of the quintessence potential-quintessence field is of exponential type.

\section{DISCUSSIONS AND FINAL REMARKS}

In the present paper we have presented the general solution of the gravitational field equations in a flat geometry for a mixture of matter and quintessence field, with the field $\phi$ as a free function. The basic physical assumption is the dependence of all parameters on the volume scale factor of the Universe. Consequently, the solution of the field equations can be represented in a parametric form. By supposing that the volume-scalar field dependence is of exponential type, an exact solution corresponding to a dust filled Universe has been obtained. The solution depends on two arbitrary numerical parameters, an integration constant and the coefficient $n$ relating the quintessence field to the volume scale factor of the Universe. The range of acceptable values of $n$ can be severely restricted by imposing the conditions of accelerated expansion and that the energy density of the $\phi$ field be of the same order of magnitude as the matter energy density.

In Fig. 1 we present the time variation of the energy density of the dust matter and of the quintessence field, for different values of $n$. The energy density of matter is practically insensitive to the value of $n$, its time variation being mainly determined by the evolution of the volume scale factor. For the chosen value of the integration constant $g$ there are two distinct periods in the evolution of the Universe. During the first phase the matter energy density dominates the scalar field energy density, $\rho_{m}>\rho_{\phi}$, but after a critical time $t_{c}$, the energy density of the quintessence field becomes larger than the energy of the cold matter and the dynamics of the Universe is determined by the scalar field. The variation of the deceleration parameter $q$, represented in Fig. 2, shows a similar evolution, with decelerating expansion during the matter domination phase and accelerated evolution for $t>t_{c}$. In the large time limit the deceleration parameter tends to $q=\frac{9}{2} n-1$, leading to an approximate range of $q \in(-1,-0.5)$. The variation of the pressure of the quintessence field, presented in Fig. 3, shows that for all times $p_{\phi}<0$ and thus a negative pressure drives the accelerate expansion of the Universe. But for larger values of $g$ the energy density of the scalar field dominates the matter energy density from the early beginning of the cosmological evolution. 


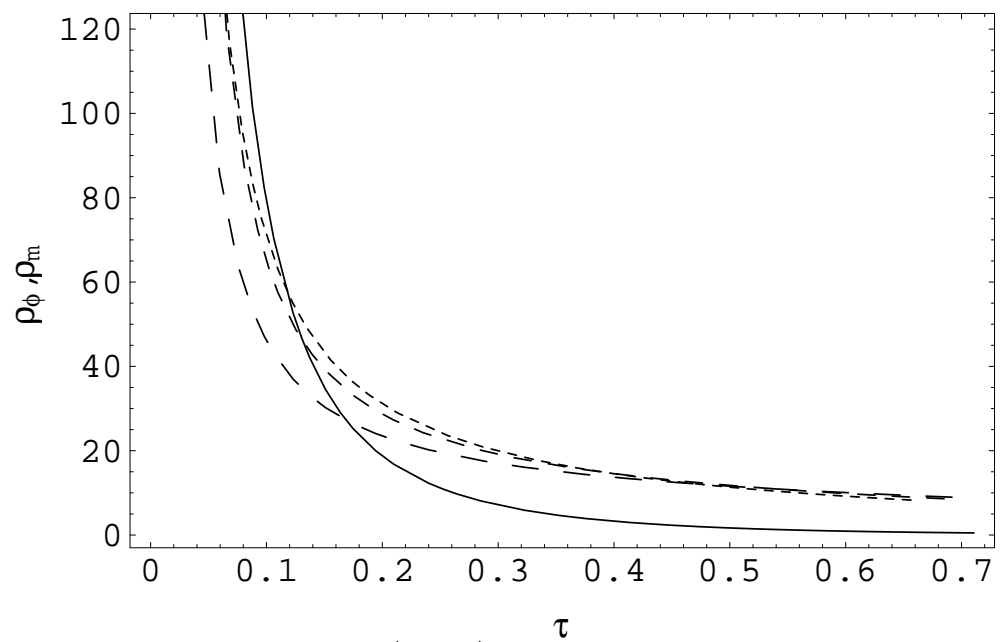

FIG. 1. Variation as a function of the time $\tau=\sqrt{\rho_{0}}\left(t-t_{0}\right)$ of the energy density of the matter $\rho_{m}$ for $n=0.11$ (solid curve) and of the quintessence field $\rho_{\phi}$ for $n=0.11$ (dotted curve), $n=0.09$ (dashed curve) and $n=0.07$ (long dashed curve) . The constant $g$ has been normalized by means of the condition $\frac{g}{\rho_{0}}=30$.

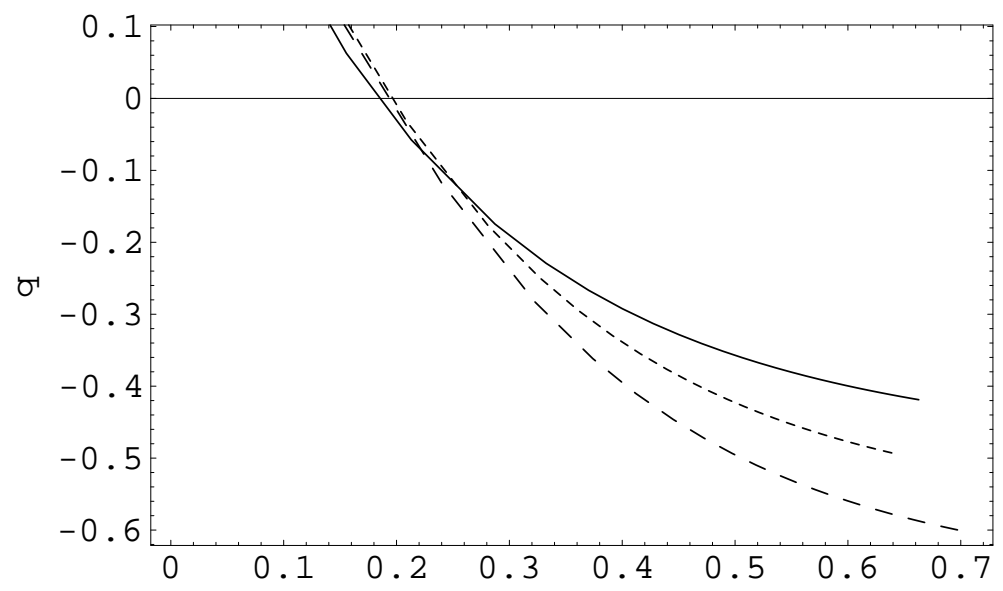

FIG. 2. Variation as a function of the time $\tau=\sqrt{\rho_{0}}\left(t-t_{0}\right)$ of the deceleration parameter $q$ for $n=0.11$ (solid curve), for $n=0.09$ (dotted curve) and for $n=0.07$ (dashed curve). The constant $g$ has been normalized by means of the condition $\frac{g}{\rho_{0}}=30$.

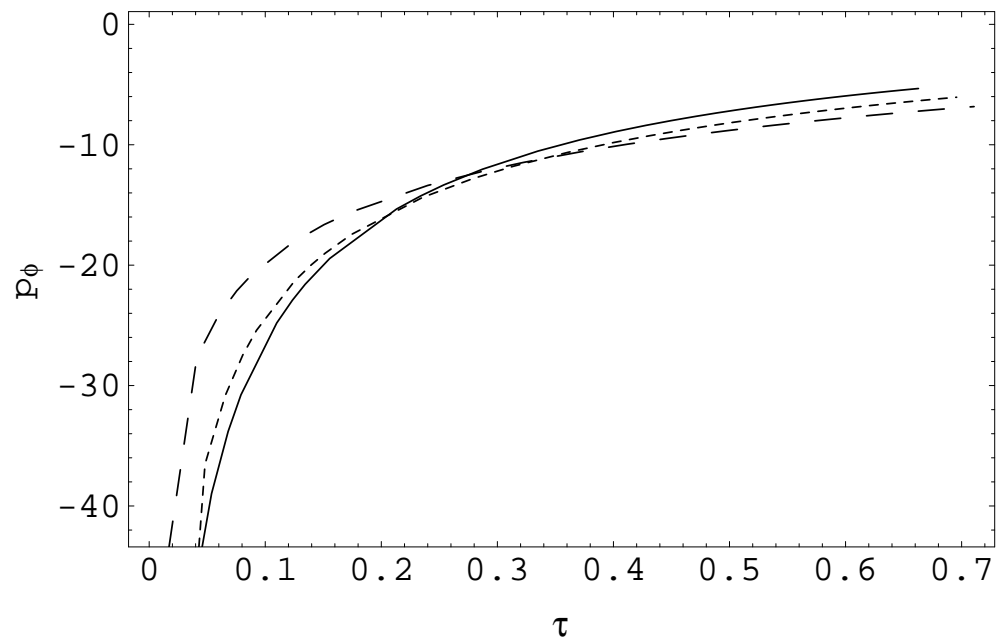


FIG. 3. Variation as a function of the time $\tau=\sqrt{\rho_{0}}\left(t-t_{0}\right)$ of the pressure $p_{\phi}$ of the quintessence field for $n=0.11$ (solid curve), for $n=0.09$ (dotted curve) and for $n=0.07$ (dashed curve). The constant $g$ has been normalized by means of the condition $\frac{g}{\rho_{0}}=30$.

In this model the evolution of the Universe is generally insensitive to the numerical values of the two constants $n$ and $g$ involved in the physical model. For all times the energy densities of matter and scalar field are of same order of magnitude. Therefore no fine-tunning of parameters is necessary to explain the present state of the Universe and the coincidence problem is also solved naturally, without imposing special initial conditions for the quintessence field or its potential.

In the limit of large time, $V \rightarrow \infty$, we obtain $V \sim t^{2 / 3 n}$ and $a \sim t^{2 / 9 n}$. In the same limit the other physical quantities behave like

$$
\begin{aligned}
\rho_{m}(t) & \sim t^{-\frac{2}{3 n}}, \rho_{\phi}(t) \sim \frac{g}{3} t^{-2}+\frac{3 \rho_{0}}{1-3 n} t^{-\frac{2}{3 n}} \\
U(t) & \sim\left(\frac{1}{3}-\frac{n}{2}\right) g t^{-2}+\frac{15 n-4}{6(1-3 n)} \rho_{0} t^{-\frac{2}{3 n}}
\end{aligned}
$$

In the large time limit the deceleration parameter is a constant.

The gravitational field equations with quintessence fields with arbitrary self-interaction potentials are of a considerable complexity even for the flat FRW geometry. Because of this mathematical complexity usually only very simple models, with a power-law dependence of the scale factor and of the scalar field, have been investigated. The more complicated models presented in this paper can maybe provide a more complete description of the early and late time behavior of our Universe. In particular these models lead to a late time non-decelerating evolution of the Universe, with numerical values of the deceleration parameter concordant to the astronomical observations [1], [2]. On the other hand the energy contribution of the quintessence scalar field $\phi$ brings the total density parameter $\Omega$ very close to the critical value $\Omega=1$.

[1] Riess A. G. et al, Astron. J. 116 (1998) 1099.

[2] Perlmutter S. et al, Astrophys. J. 517 (1999) 517.

[3] Perlmutter S. et al, Nature, 391 (1998) 51.

[4] Ostriker J. P. and Steinhardt P. J., Nature, 377 (1995) 600.

[5] Weinberg S., Rev. Mod. Phys. 61 (1989) 1; Weinberg S., astro-ph/0005265 (2000); Gasperini M., Phys. Lett. B 194 (1987) 347; Gasperini M., Class. Quantum. Grav. 5 (1988) 521.

[6] Bertolami O. and Martins P. J., Phys. Rev. D 61 (1999) 064007; Sen A. A., Sen S. and Sethi S., Phys. Rev. D 63 (2001) 107501; Sen S. and Sen A. A., Phys. Rev. D 63 (2001) 124006.

[7] Caldwell R., Dave R. and Steinhardt P. J., Phys. Rev. Lett. 80 (1998) 1582.

[8] Baccigalupi C., Matarese S. and Perrota F., Phys. Rev. D 62 (2000) 123510; Brax P., Martin J. and Riazuelo A., Phys. Rev. D 62 (2000) 103505; Chimento L. P., Jakubi A. S. and Pavon D., Phys. Rev. D 62 (2000) 063508; Matos T. and Urena-Lopez L. A., Class. Quantum Grav. 17 (2000) L75; Ng S. C. C., Phys. Lett. B 485 (2000) 1; Riazuelo A. and Uzan J.-P., Phys. Rev. D 62 (2000) 083506.

[9] Zlatov I., Wang L. and Steinhardt P. J., Phys. Rev. Lett.82 (1999) 896.

[10] Steinhardt P. J., Wang L. and Zlatev I., Phys. Rev. D 59 (1999) 123504.

[11] Sahni V. and Wang L., Phys. Rev. D 62 (2000) 103507.

[12] Urena-Lopez L. A. and Matos T., Phys. Rev. D 62 081302; Sahni V. and Starobinsky A. A. , Int. J. Mod. Phys. D 9 (2000) 373.

[13] Sen S. and Seshadri T. R., gr-qc/0007079 (2000).

[14] Saini T. D., Raychaudhuri S., Sahni V. and Starobinski A. A., Phys. Rev. Lett. 85 (2000) 1162.

[15] Faraoni V., Phys. Rev. D 62 (2000) 023504; Uzan J. P., Phys. Rev. D 59 (1999) 123510; Bertolo N. and Pietroni M., Phys. Rev. D 61 (1999) 023518; Liddle A. R. and Scherer R. J., Phys. Rev. D 59 (1998) 023509; Santos C. and Gregory R., Annals Phys. 258 (1997) 111. 